\title{
ROBUST TRACKING BASED ON BOOSTED COLOR SOFT SEGMENTATION AND ICA-R
}

\author{
Fan Yang, Huchuan Lu
}

\author{
Department of Electronic Engineering \\ Dalian University of Technology \\ Dalian, CHINA
}

\begin{abstract}
In this paper, we propose a novel approach for robust visual tracking. To separate the foreground from the background, we propose a novel Boosted Color Soft Segmentation (BCSS) algorithm and incorporate Independent Component Analysis with Reference (ICA-R) into the tracking framework. In addition, we design a scheme to fuse and update BCSS and ICA-R. We also propose adaptive scale of tracking window to handle objects' scale changes. Experiments shows that our approach is more robust than some popular tracking systems.
\end{abstract}

Index Terms - visual tracking, soft segmentation, ICA$\mathrm{R}$, adaptive scale

\section{INTRODUCTION}

Recently tracking has been formulated as a classification task to separate the target from the background. Collins and Liu [1] propose to adaptively select color features that best discriminate the object from the background. Avidan [2] uses an adaptive ensemble of classifiers combining color and texture features. Grabner et al. [3] design an Online Adaboost classifier that selects weak classifiers adaptively. Furthermore, to solve drifting problem, SemiBoost tracker [4], also a boosting classifier combined with semi-supervised learning, has been proposed.

Despite their achievements these approaches still suffer from complicated training process and drifting. They cannot handle occlusion and scale change well. So they are prone to fail in some cases.

To overcome the problem, we propose a novel tracking method that is simple and straightforward but efficient and robust. In this paper, we aim to distinguish the foreground from the background by enhancing the discrepancy between them. Motivated by soft segmentation [5] successfully applied in human detection, we propose Boosted Color Soft Segmentation, combined with Independent Component Analysis with Reference (ICA-R) [6], for human tracking. Additionally, we design a fusion strategy, an updating scheme and adaptive scale to ensure robust performance.
Yen-Wei Chen

\author{
College of Information Science and Engineering \\ Ritsumeikan University \\ Kusatsu, JAPAN
}

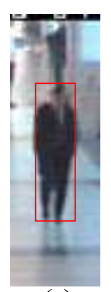

(a)

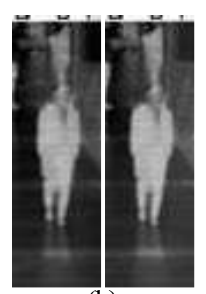

(b)

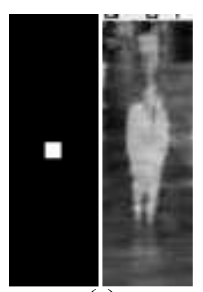

(c)
Fig. 1. Illustration of BCSS and ICA-R. (a) Initial template. (b) Result of BCSS (left) and [5] (right). (c) Reference signal (left) and result of ICA-R (right).

\section{OUR APPROACH}

To begin with, we manually draw a bounding box $s=$ $\left(h_{1}, w_{1}\right)$ enclosing the target in the first frame (see Figure 1(a)). The target with the background around the box is treated as initial template.

\subsection{Boosted Color Soft Segmentation}

"Soft segmentation" aims to segment pixels in the region into foreground and background. Given a set of RGB pixel values $\mathbf{x}_{i}=\left(x_{r}, x_{g}, x_{b}\right)$, the task of soft segmentation is to seek a linear projection of the pixel values $y=\mathbf{w}^{T} \mathbf{x}$ which maximizes the separation between foreground and background. [5] propose a simplified model by defining $\mathbf{w} \propto\left(\mathbf{m}_{2}-\mathbf{m}_{1}\right)$ where $\mathbf{m}_{1}$ and $\mathbf{m}_{2}$ are the sample means of the background and foreground classes respectively. This model reduces the computational complexity, but it is unreliable to assume that the covariance of both foreground and background distributions is isotropic. Besides, only sample means cannot comprehensively represent the color distribution of pixels. To solve the problem, we propose a Boosted Color Soft Segmentation (BCSS) algorithm.

As a successful boosting method, Adaboost [7] has been widely used in classification. Since the features we use are only RGB colors (number of weak learners $K=3$ ) whose dimension is relatively small, we simply adopt Adaboost-type method in BCSS algorithm without loss of efficiency.

The main idea of Adaboost is to collect a number of weak 


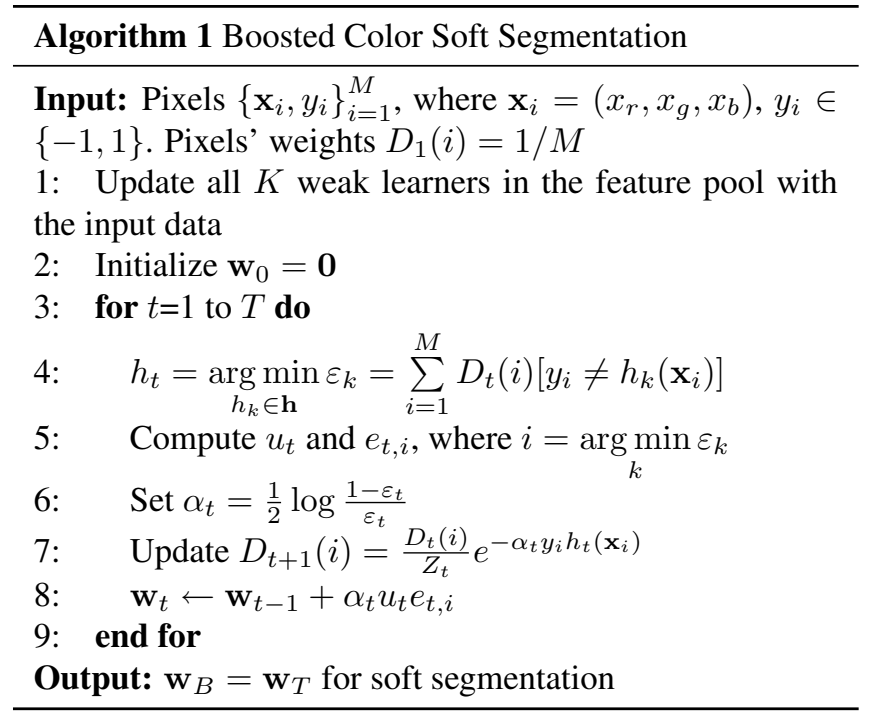

learners $h(x)$ into a strong classifier $H(x)=\operatorname{sign}\left(\sum_{t=1}^{T} \alpha_{t} h_{t}(x)\right)$. Rewriting $h_{t}(x)$, we obtain

$$
h_{t}(x)=u_{t}\left(e_{t, i}^{T} x+b_{t}\right)
$$

where $e_{t, i}^{T}$ is a vector. All elements of $e_{t, i}^{T}$ is 0 except that the $i^{\text {th }}$ element $e_{i}=1$. $b_{t}$ is linear bias and $u_{t}$ is polarity that is either -1 or 1 . Then $H(x)$ is rewritten as

$$
H(x)=\operatorname{sign}\left[\left(\sum_{t=1}^{T} \alpha_{t} u_{t} e_{t, i}\right)^{T} x+\sum_{t=1}^{T} \alpha_{t} u_{t} b_{t}\right]
$$

Let $\mathbf{w}=\sum_{t=1}^{T} \alpha_{t} u_{t} e_{t, i}$ and $\mathbf{b}=\sum_{t=1}^{T} \alpha_{t} u_{t} b_{t}$, then $H(x)=$ $\operatorname{sign}\left(\mathbf{w}^{T} \mathbf{x}+\mathbf{b}\right)$. It means that $H(x)$ is a hyperplane to separate positive and negative samples and is consistent with the goal of soft segmentation in seeking a projection space. However, different from standard Adaboost, we only record $\alpha_{t}, u_{t}$ and $e_{t, i}$ for calculating $\mathbf{w}_{B}$. After boosted selection of discriminative dimension of feature vectors, we finally obtain a hyperplane $\mathbf{w}_{B}$ with most discriminative power. The process of BCSS is shown in Algorithm 1. Note that we omit bias $b$ in our algorithm. The weak learner we use is given by

$$
h_{k}(x)=\operatorname{sign}\left[p\left(y=1 \mid f_{k}(x)\right)-p\left(y=-1 \mid f_{k}(x)\right)\right]
$$

where $p\left(f_{k}(x) \mid y=1\right)$ complies with $N\left(\mu_{1}, \sigma_{1}\right)$ and similarly for $y=-1$. In a frame, after locating the object's position, we collect new samples to update weak learners by replacing old $\mu_{1}$ and $\sigma_{1}$ of foreground and $\mu_{-1}$ and $\sigma_{-1}$ of background with new ones.

When obtaining $\mathbf{w}_{B}$, we compute $y=\mathbf{w}_{B}^{T} \mathbf{x}$ to softly segment the original frame. The segmented image is shown in Figure 1(b), compared with segmentation result of [5]. We can see that the contrast between foreground and background after BCSS is sharper than that of [5].

\subsection{ICA-R}

ICA-R is an efficient one-unit ICA method utilizing prior information [6]. It incorporates prior information about the desired source signal as a reference signal to extract only the desired source signal, which is the closest one to the reference signal, from an observed mixture of source signals. For input source signals $\mathbf{x}$ and the reference signal $\mathbf{r}$, ICA-R seek a projection space $y=\mathbf{w}_{I}^{T} \mathbf{x}$ to separate the desired signal. For tracking problem, the desired signal is the target and the mixture of signals is a frame, so we can naturally apply ICA$\mathrm{R}$. We use a fast one-unit ICA-R algorithm proposed by [6]. Its core idea is to maximize the negentropy $J(y)$ given by

$$
\begin{aligned}
& J(y) \approx \rho[E\{G(y)\}-E\{G(v)\}]^{2} \\
& \text { s.t. } \quad g(\mathbf{w}) \leq 0
\end{aligned}
$$

where $\rho$ is a positive constant, $v$ is a Gaussian variable having zero mean and unity variance. $G(x)=e^{\frac{x^{2}}{2}}$ in our experiments. $E\{x\}$ is the expectation of $x . g(\mathbf{w})=\varepsilon(y, r)-\tau \leq 0$, in which the closeness measure $\varepsilon(y, r)$ is defined to achieve its minimum when $\mathbf{w}=\mathbf{w}^{\text {opt }}$ and $\tau$ is a threshold.

To find the optimal $\mathbf{w}^{o p t}$, a Newton-like learning method is adopted to iteratively update the vector w. Details can be found in [6]. As Figure 1(c) shows, we use an image in which a small region in the center is white and all other pixels are black as the reference signal. Given a source image (see Figure 1(a)), ICA-R learns a $\mathbf{w}_{I}$ to separate the object from the background.

\subsection{Combination}

For each frame, we obtain projected images $Y_{B}$ and $Y_{I}$ using BCSS and ICA-R respectively, and normalize the pixel values of the two images to 0-1. Rather than calculating the weighted sum of $Y_{B}$ and $Y_{I}$, we convert them to probabilistic maps in which pixel values indicate its probability to be foreground. We then obtain the joint probabilistic map $P$ by calculating the joint probability distribution that is given by

$$
P=p\left(y=1 \mid Y_{B}, Y_{I}\right)
$$

$Y_{B}$ and $Y_{I}$ are independent so Equation 5 can be rewritten as

$$
P=p\left(y=1 \mid Y_{B}\right) \cdot p\left(y=1 \mid Y_{I}\right)
$$

We assume that $p\left(Y_{B} \mid y=1\right)$ and $p\left(Y_{I} \mid y=1\right)$ comply with Gaussian distribution $N\left(\mu_{B}, \sigma_{B}\right)$ and $N\left(\mu_{I}, \sigma_{I}\right)$. Thus the joint probabilistic map is defined as

$$
P(i)=e^{-\frac{\left(Y_{B}(i)-\mu_{B}\right)^{2}}{\sigma_{B}^{2}}} \cdot e^{-\frac{\left(Y_{I}(i)-\mu_{I}\right)^{2}}{\sigma_{I}^{2}}}
$$

where $i=1,2, \ldots, N, N$ is the total number of pixels in a frame. $\sigma_{B}^{2}=\sigma_{I}^{2}=0.2$ and $\mu_{B}=\mu_{I}=1$. By adopting Equation 7, the contrast between foreground and background is enhanced, as shown in Figure 2. Subsequently, we use Meanshift [8] to find the maximum in the joint probabilistic map, and regard it as the center of the target. 


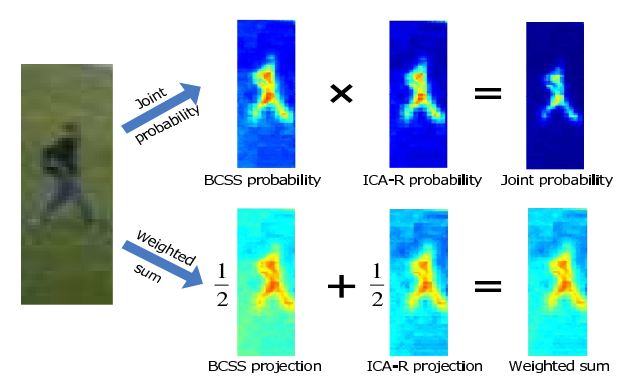

Fig. 2. Joint probabilistic map and weighted sum map.

\subsection{Update}

In our approach, we devise a scheme to online update $\mathbf{w}_{B}$ and $\mathbf{w}_{I}$. We select image patch $I_{t}$ containing the tracked object and the background in each frame $F_{t}$, resize $I_{t}$ to a constant size and accumulate all $I_{1: t}$ according to the following criteria

$$
I_{t}^{*}=\frac{I_{1}}{2^{t-1}}+\frac{I_{2}}{2^{t-1}}+\frac{I_{3}}{2^{t-2}}+\cdots+\frac{I_{t-1}}{2^{2}}+\frac{I_{t}}{2}
$$

where $I_{t}^{*}$ denotes the accumulated image patch until frame $F_{t}$. We focus on the latest frames while gradually "forgetting" old frames that cannot well represent the current target. For computational efficiency, we update $\mathbf{w}_{B}$ and $\mathbf{w}_{I}$ every $f_{B}$ and $f_{I}$ frames. For BCSS, we replace all the old weak learners with newly computed ones using $I_{t}^{*}$. Meanwhile, we treat $I_{t}^{*}$ as the new mixture of source signals, and perform ICA-R with reference signal $\mathbf{r}$. After computing the new $\mathbf{w}_{B}$ and $\mathbf{w}_{I}$, we incorporate them into the old $\mathbf{w}_{B}$ and $\mathbf{w}_{I}$ using the following equation

$$
\hat{\mathbf{w}}=\mathbf{w}+\eta \mathbf{w}_{n}
$$

where $\mathbf{w}_{n}$ is the newly computed projection vector. $\eta$ is learning rate to control the updating speed, $\eta=0.5$.

\subsection{Adaptive scale}

To handle scale changes, we devise a mechanism to achieve adaptive scale. As Figure 3 shows, if the object's size is bigger than the size of tracking window, some pixels outside the window are "bright" and close to the target but much different from the true background. Similarly, tracking window bigger than the target introduces "dark" pixels that fall into the foreground region but differ from the foreground. Based on the observation, we propose to count the number of "bright" pixels in the background region and "dark" pixels in the foreground region given by the current tracking window in each frame $F_{t}$, representing them as $N_{b g, t}$ and $N_{f g, t}$. We calculate the increment $\Delta N_{b g, t}=N_{b g, t}-N_{b g, 1}$ and $\Delta N_{f g, t}=N_{f g, t}-N_{f g, 1}$ with respect to the initial template. Then we employ the following criteria to compute the per-

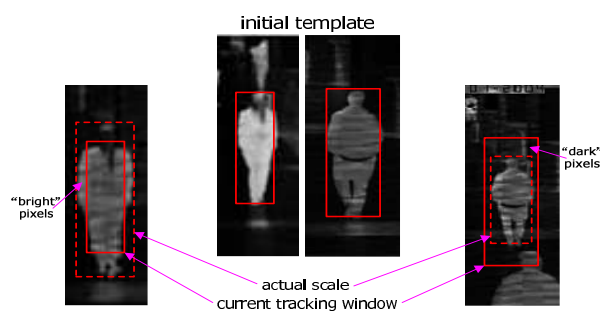

Fig. 3. Influence of objects' scale changes.

centage of change of height and width.

$$
\alpha= \begin{cases}\sqrt{1-\left|\Delta N_{f g, t}\right| /\left(h_{1} w_{1}\right)} & \Delta N_{f g, t}>\Delta N_{b g, t} \\ \sqrt{\left|\Delta N_{b g, t}\right| /\left(h_{1} w_{1}\right)+1} & \Delta N_{b g, t}>\Delta N_{f g, t}\end{cases}
$$

If $\Delta N_{f g, t}>\Delta N_{b g, t}$, the tracking window is bigger than the true target; so we shrink the window by setting $\alpha<1$. Whereas if $\Delta N_{b g, t}>\Delta N_{f g, t}$, we need to enlarge the window, so $\alpha>1$. The height and width is updated by

$$
\begin{aligned}
& h=(\alpha+\xi) h_{1} \\
& w=(\alpha+\xi) w_{1}
\end{aligned}
$$

where $\xi \propto(1-\alpha)$ is a smoothness term to avoid abrupt and inaccurate change of scale in some cases, i.e. the target is occluded by another object in the same color.

\section{EXPERIMENTS}

We test our tracking approach on public video sequences from CAVIAR dataset and PETS2001 dataset. These sequences include scale changes, simple occlusion and background clutter. We update $\mathbf{w}_{B}, \mathbf{w}_{I}$ and size of the tracking window $s=(h, w)$ every 5 frames. Note that all tracking parameters are fixed for all testing sequences. For comparison we also run Meanshift [8], Online Adaboost (OAB) [3] and SemiBoost [4]. The screenshots of results and quantitative comparison are shown in Figure 4 and Figure 5.

The first row of Figure 4 shows results on sequence "camera1" from PETS2001. Only Meanshift fails at the beginning while the other three methods successfully locate the person during the entire tracking procedure. However, we use simple RGB features, compared with Haar-like wavelet used in $\mathrm{OAB}$ and SemiBoost. The other three sequences are all from CAVIAR dataset. In "OneStopEnter2cor" (2nd row), although all methods keep track of the person, our approach is more accurate due to the adaptive scale strategy. In "ThreePastShop2cor" (3rd row), Meanshift and SemiBoost lose the object when simple occlusion occurs, while OAB yields inaccurate result owing to its constant tracking window. The two sequences demonstrate that our approach can easily handle scale changes. When the target's scale enlarges or shrinks, the tracking window can adjust its size accordingly. In "ShopAssistant2cor" (4th row), a person walks along the passage and 


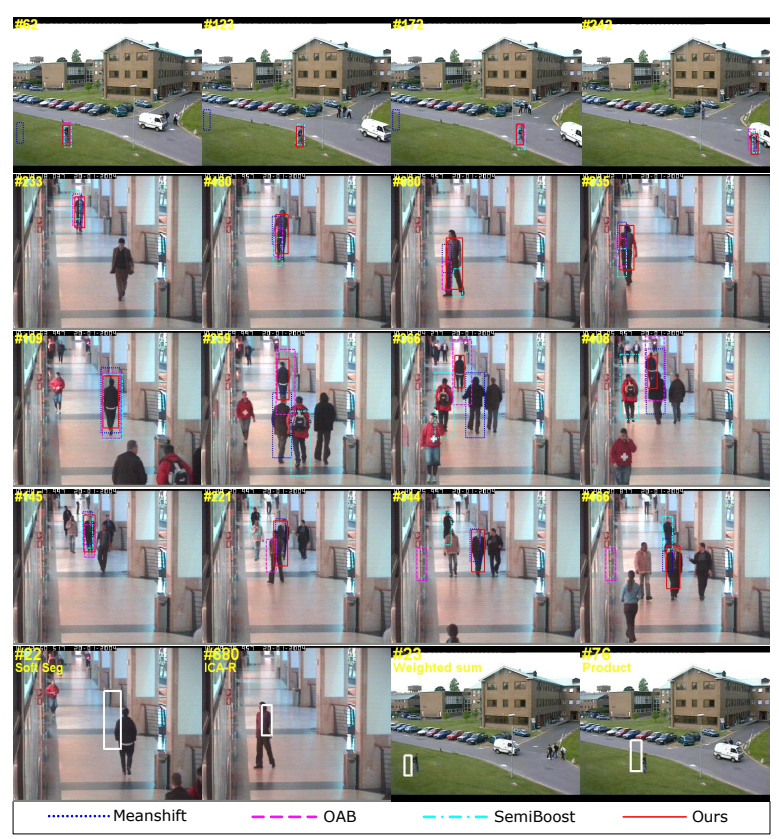

Fig. 4. Screenshots of tracking results.

is occluded by another person. Our approach succeeds in locating the target. In contrast, OAB and SemiBoost suffer from drifting, and Meanshift cannot accurately find the center of the target.

Moreover, only BCSS or ICA-R cannot guarantee stable tracking results, as shown in the bottom row (the first two images) in Figure 4. Note that the update scheme and adaptive scale adjustment remain unchanged. Only using BCSS leads the tracker easily drift away; only using ICA-R makes the results also inaccurate. So the poor performance of individual algorithms motives us to fuse them together. We also show results of using other fusing strategies in the bottom row (the last two images) in Figure 4. We can see that if we use weighted sum or product to combine the two probabilistic maps, the tracker will also lose the object. Therefore, our fusing strategy of computing joint probability distribution outperforms the other two fusing methods.

Experiments demonstrate that our approach is robust in handling short-time occlusion and scale changes, although it is conceptually simple and straightforward.

\section{CONCLUSIONS}

In this paper, we propose a robust tracking framework by fusing Boosted Color Soft Segmentation and ICA-R. The contribution of this paper is four-fold: (1) We apply ICA-R that is mainly used for speech analysis to the tracking problem. (2) We devise a new soft segmentation approach by boosting strategy with online updating. (3) We adaptively fuse the results of ICA-R and BCSS into a joint probabilistic map. (4)
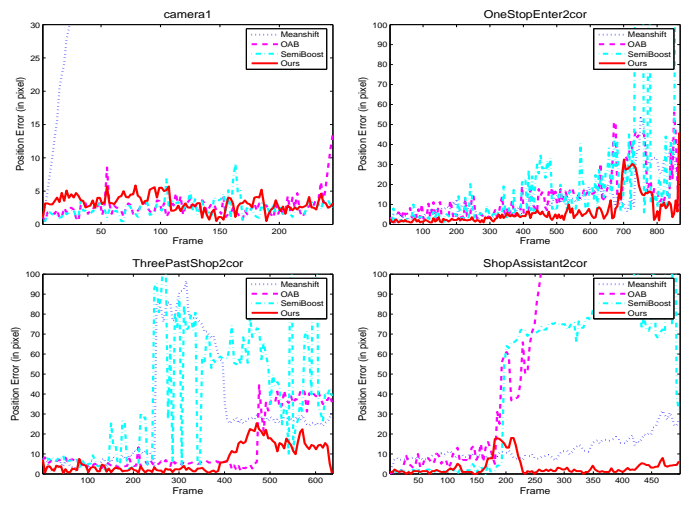

Fig. 5. Error plots of different approaches on testing sequences. The vertical axis indicates the error of the central position of the object, compared with groundtruth.

We also devise an adaptive scale scheme to handle the object's scale changes. Experiments show that our method is more robust in comparison with Meanshift, OAB and SemiBoost.

\section{REFERENCES}

[1] R.T. Collins, Y. Liu, and M. Leordeanu, "Online selection of discriminative tracking features," IEEE Transactions on Pattern Analysis and Machine Intelligence, pp. 16311643, 2005.

[2] S. Avidan, "Ensemble tracking," in CVPR, 2005, vol. 2, pp. 494-501.

[3] H. Grabner and H. Bischof, "On-line boosting and vision," in CVPR, 2006, vol. 1, pp. 260-267.

[4] H. Grabner, C. Leistner, and H. Bischof, "Semisupervised on-line boosting for robust tracking," in ECCV, 2008, vol. 2, pp. 234-247.

[5] Patrick Ott and Mark Everingham, "Implicit color segmentation features for pedestrian and object detection," in ICCV, 2009.

[6] Q.H. Lin, Y.R. Zheng, F.L. Yin, H. Liang, and V.D. Calhoun, "A fast algorithm for one-unit ICA-R," Information Sciences, vol. 177, no. 5, pp. 1265-1275, 2007.

[7] Y. Freund and R.E. Schapire, "A decision-theoretic generalization of on-line learning and an application to boosting," Journal of computer and system sciences, vol. 55, no. 1, pp. 119-139, 1997.

[8] D. Comaniciu, V. Ramesh, and P. Meer, "Kernel-based object tracking," IEEE Transactions on Pattern Analysis and Machine Intelligence, vol. 25, no. 5, pp. 564-577, 2003. 